\title{
Magnetic frustration and spontaneous rotational symmetry breaking in $\mathrm{PdCrO}_{2}$
}

\author{
Dan Sun, ${ }^{1,2,{ }^{*}}$ Dmitry A. Sokolov, ${ }^{1, *}$ Jack M. Bartlett, ${ }^{1,3}$ Jhuma Sannigrahi, ${ }^{1}$ Seunghyun Khim, ${ }^{1}$ Pallavi Kushwaha, ${ }^{1,4}$ \\ Dmitry D. Khalyavin, ${ }^{5}$ Pascal Manuel, ${ }^{5}$ Alexandra S. Gibbs, ${ }^{5}$ Hidenori Takagi,,${ }^{6,7,8}$ Andrew P. Mackenzie, ${ }^{1,3, \dagger}$ \\ and Clifford W. Hicks ${ }^{1, ٪}$ \\ ${ }^{1}$ Max Planck Institute for Chemical Physics of Solids, Nöthnitzer Strasse 40, 01187 Dresden, Germany \\ ${ }^{2}$ Los Alamos National Laboratory, Los Alamos, New Mexico 87545, USA \\ ${ }^{3}$ SUPA, School of Physics and Astronomy, University of St. Andrews, St. Andrews KY16 9SS, United Kingdom \\ ${ }^{4}$ CSIR- National Physical Laboratory, Dr. K. S. Krishnan Marg, New Delhi 110012, India \\ ${ }^{5}$ ISIS Facility, STFC Rutherford Appleton Laboratory, Chilton, Didcot OX11 0QX, United Kingdom \\ ${ }^{6}$ Max Planck Institute for Solid State Research, Heisenbergstrasse 1, 70569 Stuttgart, Germany \\ ${ }^{7}$ Institute for Functional Matter and Quantum Technologies, University of Stuttgart, Pfaffenwaldring 57, 70569 Stuttgart, Germany \\ ${ }^{8}$ Department of Physics, The University of Tokyo, 7-3-1 Hongo, Bunkyo-ku, Tokyo 113-0022, Japan
}

(Received 16 May 2019; revised manuscript received 19 August 2019; published 10 September 2019)

\begin{abstract}
In the triangular layered magnet $\mathrm{PdCrO}_{2}$ the intralayer magnetic interactions are strong; however, the lattice structure frustrates interlayer interactions. In spite of this, long-range, $120^{\circ}$ antiferromagnetic order condenses at $T_{N}=38 \mathrm{~K}$. We show here through neutron scattering measurements under in-plane uniaxial stress and inplane magnetic field that this occurs through a spontaneous breaking of the threefold rotational symmetry of the nonmagnetic lattice, which relieves the interlayer frustration. We also show through resistivity measurements that uniaxial stress can suppress thermal magnetic disorder within the antiferromagnetic phase.
\end{abstract}

DOI: 10.1103/PhysRevB.100.094414

\section{INTRODUCTION AND BACKGROUND}

At $24 \mathrm{~K}$, solid oxygen undergoes a simultaneous Néel transition and rhombohedral to monoclinic structural transition [1,2]. The structural transition is driven by magnetic frustration: the monoclinic distortion introduces a preferred direction that relieves interlayer frustration [3]. The delafossite compound $\mathrm{PdCrO}_{2}$ is also a rhombohedral system with interlayer magnetic frustration. The $\mathrm{Cr}$ sites in each layer are triangularly coordinated and host $S=\frac{3}{2}$ spins that start to arrange themselves into short-range, $120^{\circ}$ antiferromagnetic order at 200-300 K [4-7]. However, the interlayer coupling is frustrated: in the delafossite lattice structure, the sites in each layer are centered between sites in adjacent layers. (See Fig. 1 for an illustration.) In other words, each $\mathrm{Cr}$ site is equidistant from three $\mathrm{Cr}$ sites in each adjacent layer, and the interlayer nearest-neighbor exchange coupling sums to zero. As the temperature is reduced to just above $T_{N}=$ $38 \mathrm{~K}$, the in-plane correlation length grows to $\sim 20$ lattice spacings, without appearance of interlayer correlation [8]. Then at $T_{N}$ the layers lock together to form long-range order $[5,8,9]$.

By analogy with solid oxygen, long-range order may emerge through a spontaneous rotational symmetry breaking and associated structural distortion that relieves interlayer frustration. The magnetic order of another Cr-based delafossite compound, $\mathrm{CuCrO}_{2}$, also breaks the threefold rotational

\footnotetext{
*These authors contributed equally to this work.

$\dagger$ mackenzie@cpfs.mpg.de

†hicks@cpfs.mpg.de
}

symmetry of the nonmagnetic lattice: the spin plane contains the $c$ axis, and its in-plane orientation breaks the threefold rotational symmetry [10-13]. $\mathrm{CuCrO}_{2}$ is electrically insulating, and a demonstration of this spontaneous symmetry breaking is provided by the fact that the domains can be polarized, resulting in a bulk ferroelectric polarization [10,14]. Ferroelectric polarization is also seen in another insulating delafossite, $\mathrm{AgCrO}_{2}$, showing that it too has domains [14]. In $\mathrm{CuCrO}_{2}$, the associated structural distortion is strong enough to be detected in ultrasound measurements [15]. However, so far no structural distortion has been detected in $\mathrm{PdCrO}_{2}$ $[6,16]$.

It is an important point to resolve to understand the mechanisms by which magnetic order condenses on frustrated lattices. $\mathrm{PdCrO}_{2}$ is in general an appealing target for study of magnetic order and fluctuations, because it can be grown to exceptionally high cleanliness [17]. Comparison with $\mathrm{CuCrO}_{2}$ is suggestive but not conclusive: the magnetism of the two systems is not identical, and $\mathrm{PdCrO}_{2}$ is metallic, while $\mathrm{CuCrO}_{2}$ is insulating. Instead of testing for potentially very small structural distortions, we take here the approach of using symmetry-breaking fields to polarize domains, if they are present. This is analogous to stress detwinning of compounds with nematic transitions in Ref. [18], for example. To observe domain polarization, we employ neutron scattering measurements under in-plane uniaxial stress and magnetic field. We also employ resistivity measurements: in $\mathrm{PdCrO}_{2}$ the $\mathrm{CrO}_{2}$ layers are Mott insulating but are interleaved with highly conducting Pd sheets [19].

In this work, we show that the magnetism of $\mathrm{PdCrO}_{2}$ does indeed lift the threefold rotational symmetry of the nonmagnetic lattice. Neutron scattering data indicate that the 


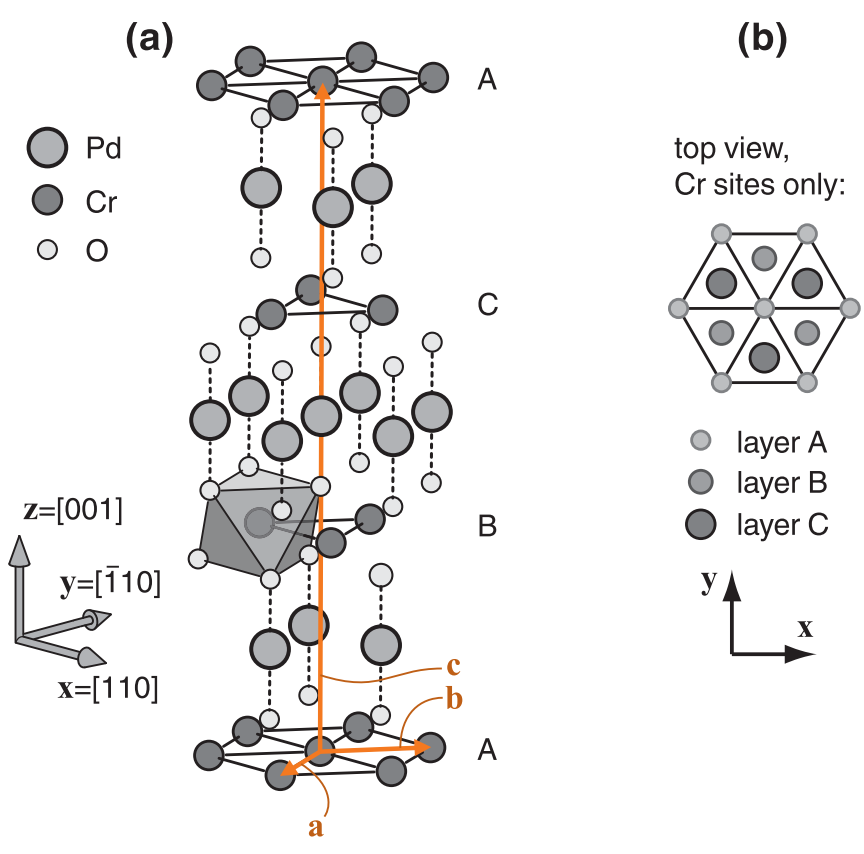

FIG. 1. (a) The delafossite structure. We take $\hat{\mathbf{x}}$ to be along the [110] direction, $\hat{\mathbf{y}}$ to be along the [110] direction, and $\hat{\mathbf{z}}$ to be along the [001] direction. In the neutron measurements, field or pressure was applied along the $y$ axis, and the scattering plane was the $x z$, i.e., the (110), plane. (b) A top view illustrating the ABCABC stacking of the layers: in this illustration, B-layer $\mathrm{Cr}$ sites are centered on the downward-pointing triangles in the A layer, and C-layer sites are centered on the upward-pointing triangles. Each $\mathrm{Cr}$ site is equidistant from the three nearest sites in adjacent layers, leading to interlayer frustration.

magnetic structure can be polarized through application of small in-plane uniaxial stress or in-plane magnetic field. The resistivity within a single domain, however, turns out to be nearly isotropic, so this domain polarization is not directly observable in resistivity data. Instead, the resistivity data reveal that uniaxial stress suppresses thermal magnetic disorder.

In Fig. 1(a) we illustrate the nonmagnetic lattice. The nonmagnetic unit cell contains three layers: the offset of the Cr layers with respect to each other introduces an ABCABC stacking. This stacking is illustrated in Fig. 1(b), a view from the top showing the relative positions of the $\mathrm{Cr}$ sites in the three layers.

The onset of $120^{\circ}$ antiferromagnetic order introduces a $\sqrt{3} \times \sqrt{3}$ reconstruction of the nonmagnetic Fermi surface. The magnetic order has been directly observed in neutron data $[4,8,16]$, and the associated electronic reconstruction is observed in quantum oscillation [5,20] and angle-resolved photoemission [19,21] data. Early signs of the reconstruction appear at $\sim 60 \mathrm{~K}[22]$, and when long-range order appears at $T_{N}$, the $c$-axis transport becomes coherent [23].

In addition to the in-plane $\sqrt{3} \times \sqrt{3}$ reconstruction, the neutron data indicate a double- $k$ interlayer order (where $k$ is a propagation vector of the magnetic structure) due to coexisting ferroic and antiferroic interlayer correlations. The study of Takatsu et al. [16] found three models that give good fits to observed neutron scattering intensities. One of them (model 2 in that study) is a mixture of two single- $k$ magnetic phases that are not related by symmetry. In this model the moment varies strongly from site to site, which is not expected with strong on-site Hund's-rule coupling that fully aligns the local electron spins. This model is therefore unlikely to be realized. The strong resistivity anisotropy of $\mathrm{PdCrO}_{2}$ is a consequence of the Mott insulating behavior of the $\mathrm{CrO}_{2}$ layer, which in turn is a consequence of the on-site alignment of spins.

The other two models (models 3 and 4 of Ref. [16]) incorporate the two $k$ 's through alternating helicity. That is, the direction of rotation of the spins on moving from site to site alternates from layer to layer. In both models, the threefold rotational symmetry of the lattice is broken, implying an associated monoclinic or triclinic lattice distortion and the presence of domains; in Ref. [16] it was assumed for comparison of observed and calculated reflection intensities that each of the three domain types was equally populated. Models 3 and 4 are closely related, differing by modest collective spin rotations, and we illustrate in Fig. 2(a) a magnetic structure that is a simplified version of both. In this simplified model the spins are all co-planar [24]. It lifts rotational symmetry in the same way as models 3 and 4 and gives only a marginally worse match to the reflection intensities reported in Ref. [16] (we quantify this statement later). Therefore for discussion we refer to this structure for now and explain possible refinements later.

The rotational symmetry breaking appears in two aspects of the structure. (1) The spins lie in the $y z$, that is, the (110) plane. (We take $\hat{\mathbf{x}} \|$ [110], $\hat{\mathbf{y}} \|$ [110], and $\hat{\mathbf{z}} \|$ [001]; see Figs. 1 and 2 for illustrations.) (2) From plane to plane the order shifts along the $y$ axis, i.e., the [1110] direction, as illustrated by the blue dashed line in Fig. 2(a). The magnetic order could equivalently condense with the spins lying in the (100) or (010) planes and shifting along, respectively, the [1 $\overline{2} 0]$ and [210] directions from plane to plane. These planes and directions are illustrated in Fig. 2. We will refer to the three domain types as (110), (100), and (010).

\section{RESULTS: NEUTRON SCATTERING}

All data reported here- - both neutron and resistivity dataare on crystals grown by the $\mathrm{NaCl}$ flux method in a sealed quartz tube, as reported in [25]. Three samples, labeled A, B, and $\mathrm{C}$, were studied with neutrons. Samples A and B were cut and polished to respective dimensions of $1.65 \times 0.97 \times 0.11$ and $2.13 \times 1.30 \times 0.07 \mathrm{~mm}^{3}$ and mounted into holders that provide the necessary mechanical protection to apply in-plane stresses to platelike samples. Force was applied using a mechanical spring which was adjusted at room temperature, so samples were cooled under nearly constant stress. The cooling rate was rapid: $\sim 20 \mathrm{~K} / \mathrm{min}$. A photograph of sample $\mathrm{A}$ is shown in Fig. 3(f). Most of the sample is exposed and experiences the full applied force; however, the neutrons do also penetrate into the ends of the sample, which are embedded in epoxy and are under lower stress than the central portion. Sample A was probed under compressive stresses of up to $\sigma=-108 \mathrm{MPa}$, and sample $\mathrm{B}$ was probed under a tensile stress of $+44 \mathrm{MPa}$. (We use negative and positive values to indicate compression and tension, respectively.) Force was applied along the [110] direction, and the scattering plane was 

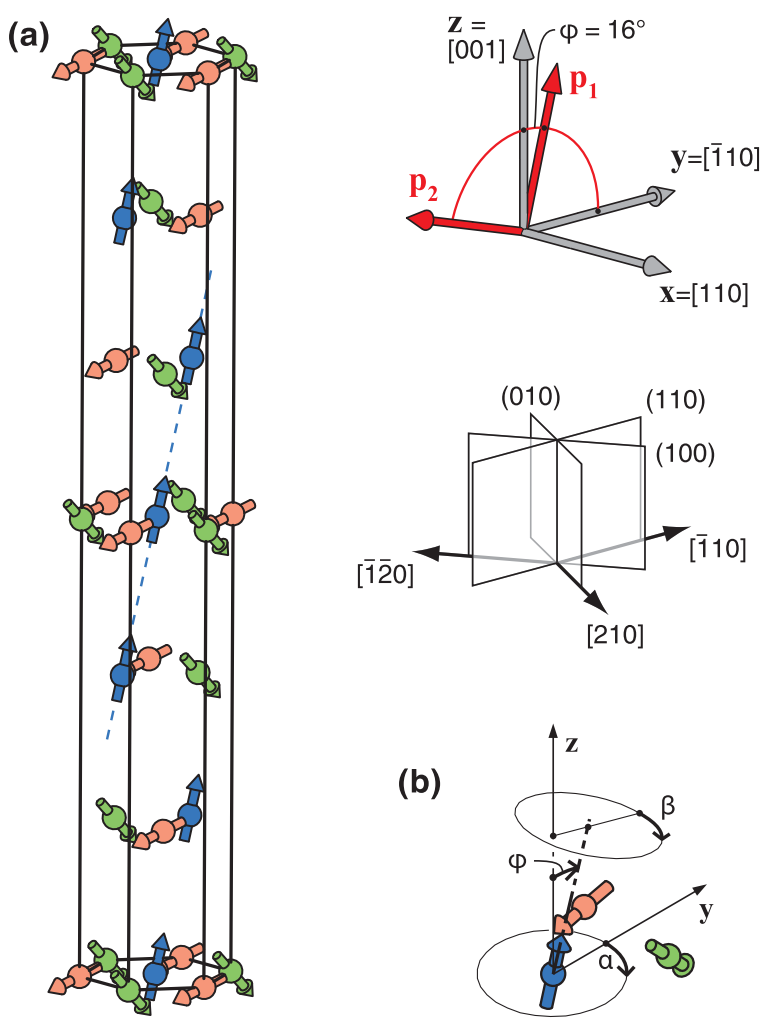

(b)

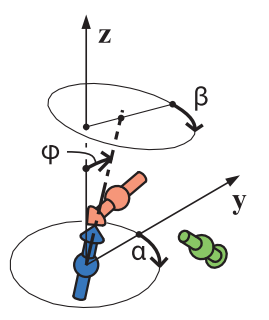

FIG. 2. (a) A magnetic structure that gives a good fit to the 58 scattering intensities reported in Ref. [16]. Only the $\mathrm{Cr}$ sites are shown. The spins are colored by orientation, and the alternating helicity is indicated by reversal of the relative positions of the green and pink spins from layer to layer. The illustrated structure is a (110) domain, meaning that the spins lie in the (110) plane. In the absence of an orienting fields, there will also be (100) and (010) domains; see the illustration of these planes to the right of the magnetic structure. Within the 18-site magnetic unit cell, it can be seen that for this domain the order shifts along the [1110] direction, i.e., the $y$ axis, from layer to layer. This interlayer ordering axis is indicated by the dashed blue line. Along this axis, spin components parallel to the vector $\mathbf{p}_{\mathbf{1}}$ are ferroically ordered from layer to layer, while components parallel to $\mathbf{p}_{\mathbf{2}}$ are antiferroically ordered. (b) Parameters for describing collective spin rotations. $\phi$ and $\alpha$ are the polar and azimuthal angles of the blue spin. $\beta$ is the angle of rotation of the spin plane about the axis of the blue spin, which sets the orientations of the other two spins. The illustrated structure has $\phi=16^{\circ}$, and $\alpha=\beta=0$.

the (110) plane, i.e., the $x z$ plane. Sample C was studied under magnetic field applied along [1110]. All neutron measurements were performed using the WISH diffractometer at the ISIS spallation neutron source. Further details of the uniaxial stress setup are given in the Appendix.

Results from samples A and B are shown in Fig. 3. At zero stress, scattering peaks appear at $(h k l)=\left(\frac{1}{3} \frac{1}{3} l\right)$ real lattice units (r.l.u.) for every half-integer $l$. The reflections are referenced to the three-layer nonmagnetic unit cell indicated in Fig. 1(a), so a two-layer periodicity, for example, yields a reflection at $l=\frac{3}{2}$. The intensities of the reflections at $l=0, \frac{3}{2}$, and 3 decreased as compressive stress was applied: their intensities at $-24 \mathrm{MPa}$ were lower, relative to the other peaks, than at $0 \mathrm{MPa}$, and they disappeared
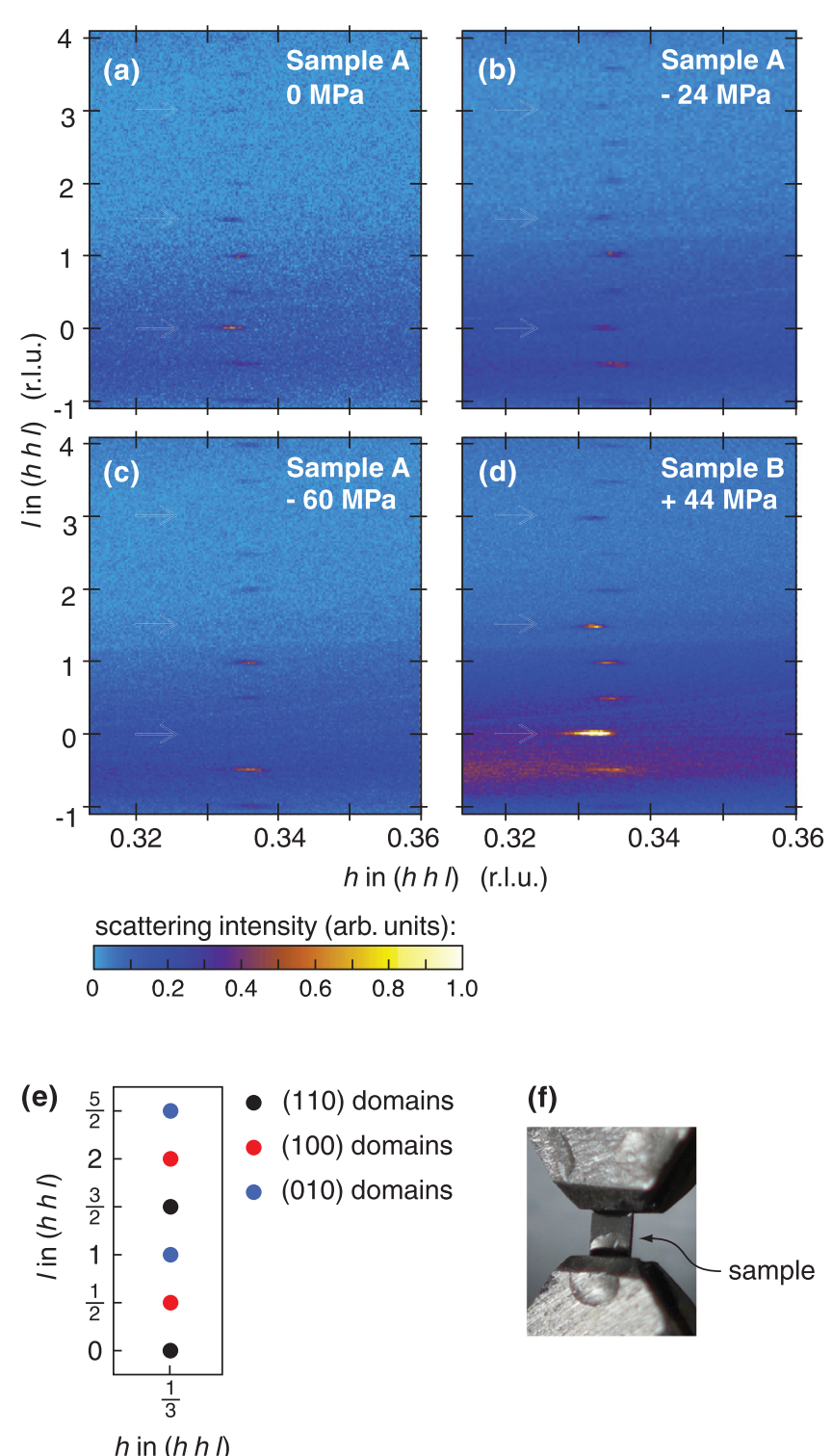

FIG. 3. (a)-(d) Magnetic reflections at $2 \mathrm{~K}$ under various uniaxial stresses $\sigma$. Stress was applied along the [110] direction, i.e., the $y$ axis in Figs. 1 and 2. The scattering vector $(h k l)=\left(\frac{1}{3} \frac{1}{3} 0\right)$ lies along the $+x$ axis. Negative values of $\sigma$ indicate compression. The intensities of the $l=0, \frac{3}{2}$, and 3 reflections evolve together with stress, which indicates that these come from a single domain type. These reflections become weaker under compressive stress and stronger under tensile stress. (e) Assignment of the reflections to magnetic domains. (f) A photograph of sample A; it is $0.97 \mathrm{~mm}$ wide and $0.11 \mathrm{~mm}$ thick.

almost entirely by-60 MPa. Conversely, under tensile stress [Fig. 3(d)] the intensities of the $l=\left\{0, \frac{3}{2}, 3, \ldots\right\}$ reflections increased while the intensities of the other peaks were reduced.

The strains generated by these stresses are very small: for a typical Young's modulus for an oxide material of $\sim 150 \mathrm{GPa}$ [26,27], stress $\sigma=60 \mathrm{MPa}$ corresponds to strain $\varepsilon \sim 4 \times$ $10^{-4}$. The drastic changes observed in reflection intensities is therefore much more likely to be due to polarization of domains than changes in the intrinsic magnetic structure. 
Under this interpretation, there are three domain types, which for $(h k)=\left(\frac{1}{3} \frac{1}{3}\right)$ give reflections at $l=\left\{0, \frac{3}{2}, 3, \ldots\right\}$, $\left\{\frac{1}{2}, 2, \frac{7}{2}, \ldots\right\}$, and $\left\{1, \frac{5}{2}, 4, \ldots\right\}$.

This is as expected for the magnetic structure shown in Fig. 2, which is derived from the reflection intensities reported in Ref. [16]. The illustrated structure is from a (110) domain, meaning that the spins lie in the (110) plane. As explained above, there is an interlayer ordering axis that is tilted away from the $c$ axis; in this domain, the magnetic order shifts along the [110] direction from plane to plane. The alternating helicity means that spin components parallel to a vector $\mathbf{p}_{\mathbf{1}}$, illustrated in Fig. 2(a), are ferroically ordered along this axis, while components along $\mathbf{p}_{\mathbf{2}} \perp \mathbf{p}_{\mathbf{1}}$ are antiferroically ordered. $\mathbf{p}_{1}$ turns out to lie, within the precision of the intensities reported in Ref. [16], along the interlayer ordering axis, in other words along a line that connects $\mathrm{Cr}$ sites in adjacent layers. The ferroically ordered components give reflections at $l=\{0,3, \ldots\}$, and the antiferroically ordered components at $l=\left\{-\frac{3}{2}, \frac{3}{2}, \ldots\right\}$.

In our scattering geometry the vector $(h k l)=\left(\frac{1}{3} \frac{1}{3} 0\right)$ lies along the $+x$, i.e., the [110], direction. For (110) domains, this is perpendicular to the interlayer ordering axis, which tilts away from the $c$ axis towards [1110]. However, for (100) and (010) domains, the interlayer ordering axis is not perpendicular to $\left(\frac{1}{3} \frac{1}{3} 0\right)$, which causes a shift of the reflections from these domains along the $l$ axis. The (100) domains give reflections at $l=\left\{\frac{1}{2}, 2, \ldots\right\}$, and (010) domains give reflections at $l=\left\{1, \frac{5}{2}, \ldots\right\}$. The $(100)$ and (010) domains are symmetrically equivalent under $y$-axis uniaxial stress and so would be suppressed or favored together, as observed. We note that a similar analysis could be done for $(h k)=\left(\frac{1}{3}-\frac{2}{3}\right)$ and $\left(-\frac{2}{3} \frac{1}{3}\right)$; however, these reflections were not accessible in this measurement.

Data under magnetic field are shown in Figs. 4(a) and 4(b). These data were collected at $T=1.5 \mathrm{~K}$, without thermal cycling between the different fields. A 13-T field applied along [110] suppresses the reflection at $\left(\frac{1}{3} \frac{1}{3} 0\right)$ completely, while the reflection at $\left(\frac{1}{3} \frac{1}{3} 1\right)$ remains. Evidently, applied field along [110] favors, like compression along this direction, the (100) and (010) domains. There is a simple explanation: for (110) domains, the [1110] direction lies within the spin plane, so for fields along this direction the magnetic susceptibility is low. For (100) and (010) domains, the paramagnetic polarization is stronger because spins tilt out of their ordering plane. In-plane magnetic fields polarize the magnetic domains of $\mathrm{CuCrO}_{2}$ by the same mechanism $[10,11,13]$.

Figure 4(e) shows integrated scattering intensities as a function of temperature for $\sigma=-60 \mathrm{MPa}$. The $l=0$ peak was the most intense at $\sigma=0$, while under $\sigma=-60 \mathrm{MPa}$ it remains suppressed up to $T_{N}$. If, at higher temperatures, there were thermal excitation of the disfavored domain, then we would expect to see some increase of the $\left(\frac{1}{3} \frac{1}{3} 0\right)$ intensity. This is not observed, and we therefore conclude that the magnetic structure remains fully polarized up to $T_{N}$. Stated more precisely, there is a first-order transition line along $\sigma=$ 0 corresponding to reorientation of the magnetic structure, and within resolution this line extends up to $T_{N}$. This point becomes important in regards to resistivity data: the intrinsic resistivity within a domain turns out to be highly isotropic, and
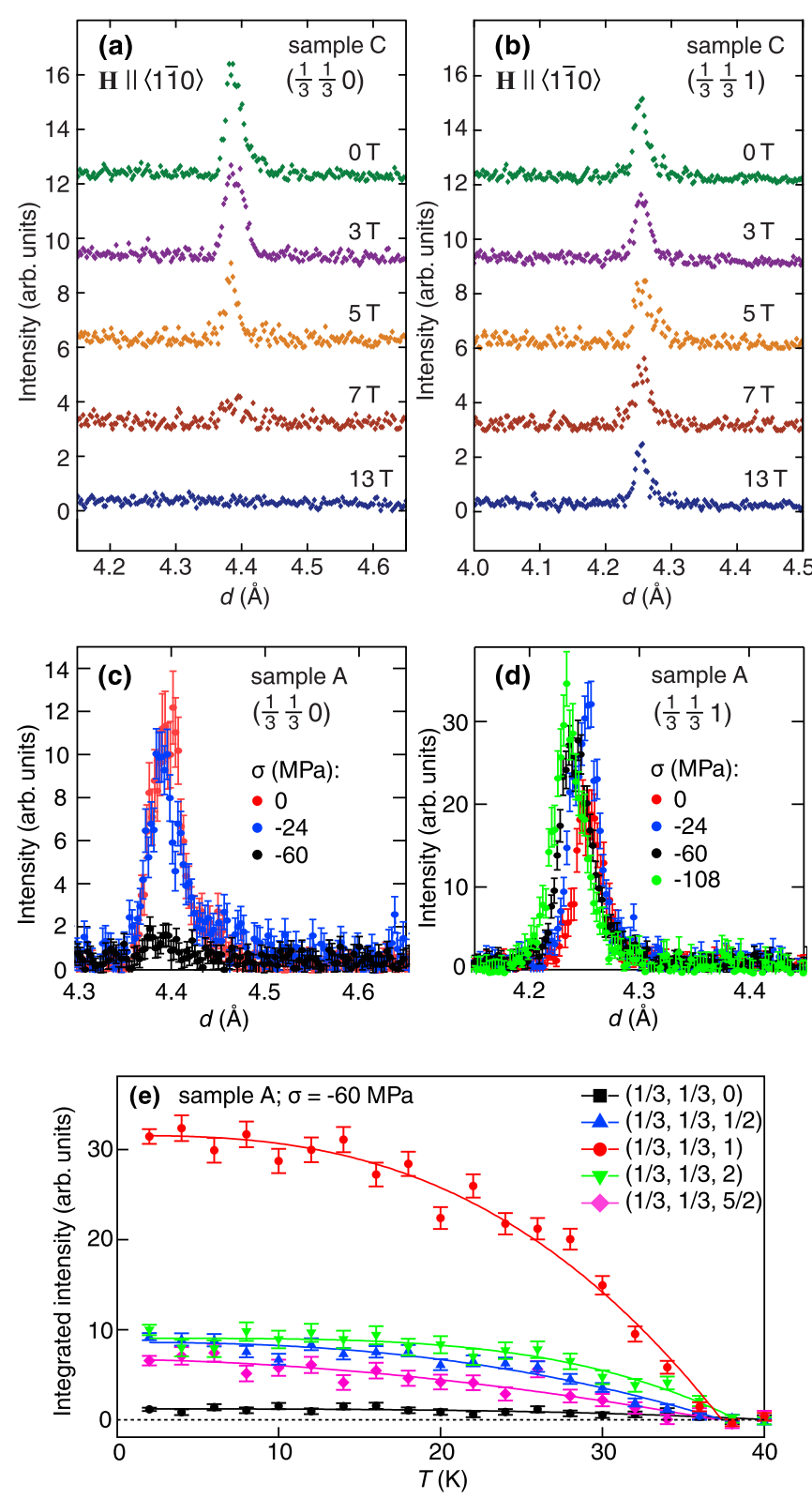

FIG. 4. (a) and (b) Field dependence of the scattering intensities at $\left(\frac{1}{3} \frac{1}{3} 0\right)$ and $\left(\frac{1}{3} \frac{1}{3} 1\right)$ for sample $\mathrm{C}$ at $1.5 \mathrm{~K}$. Field is applied along (110), i.e., the $y$ axis in Fig. 1. $d$ is the real-space periodicity. (c) and (d) Stress dependence of the magnetic scattering intensities at $\left(\frac{1}{3} \frac{1}{3} 0\right)$ and $\left(\frac{1}{3} \frac{1}{3} 1\right)$ of sample A at $2 \mathrm{~K}$. (e) Temperature dependence of the integrated intensities of various reflections under $\sigma=-60 \mathrm{MPa}$. A temperature-independent background is subtracted from each scan. Lines are guides to the eye.

this reorientation transition, although present, is not directly detectable in resistivity data.

\section{RESULTS: RESISTIVITY}

Through Cr-Pd coupling, magnetic disorder in the $\mathrm{CrO}_{2}$ layers contributes to the electrical resistivity. Comparison with a nonmagnetic analog, $\mathrm{PdCoO}_{2}$, indicates that for temperatures below $\sim 300 \mathrm{~K}$, magnetic scattering is the largest component of the inelastic part of the resistivity [4,5]. Apart 
from the effects of magnetic reconstruction, $\mathrm{PdCoO}_{2}$ has, to remarkably high precision, the same Fermi surface and Fermi velocities as $\mathrm{PdCrO}_{2}$ [5]. Therefore resistivity can be used as a sensitive probe of magnetic disorder.

Given the clear demonstration from neutron scattering data that reorientation of the magnetic structure remains a firstorder transition up to $T_{N}$, it is reasonable to hypothesize that resistivity should show a steplike feature across $\sigma=0$, corresponding to the reorientation and the intrinsic resistive anisotropy within a single magnetic domain. Resistivity anisotropy might appear more strongly in the inelastic than the elastic component because angle-resolved photoemission spectroscopy (ARPES) data indicate that the Fermi surface remains highly symmetric below $T_{N}[21,28]$. (Although the samples will not have been detwinned in the ARPES studies, the observed Fermi surfaces remain sharp and highly isotropic modulo threefold rotation symmetry.)

To measure $\rho(\varepsilon, T)$ we prepared the samples as beams and mounted them into piezoelectric-based uniaxial stress cells, as was described previously [29-31]. Far higher strains could be applied than in the neutron scattering portion of this study. A technical difference between the neutron and resistivity data is that in the former the controlled variable is stress, while in the latter it is strain. This is because for the neutron measurements force was applied using springs with spring constants much lower than those of the samples, while for the resistivity measurements piezoelectric actuators with a very high combined spring constant were used. The proportionality constant between uniaxial stress and strain is Young's modulus. We report data from three samples, two oriented along a $\langle 1 \overline{1} 0\rangle$ direction, i.e., bisecting $\mathrm{Cr}-\mathrm{Cr}$ bond directions, and one along a $\langle 100\rangle$ direction, i.e., along a $\mathrm{Cr}-\mathrm{Cr}$ bond direction. Results from two of these samples are shown in Fig. 5. Photographs of these two samples and results from the third sample are shown in the Appendix.

For both orientations, $\rho(\varepsilon)$ at $9 \mathrm{~K}$ has a relatively sharp peak, and a capacitive displacement sensor built into the apparatus indicates that at the peak $|\varepsilon|<10^{-3}$. We therefore fix $\varepsilon=0$ as the location of the peak in $\rho(\varepsilon)$ at $9 \mathrm{~K}$. This assignment is further supported by the appearance of a hysteresis loop at lower temperatures centered on this strain; larger hysteresis in domain reorientation is expected at low temperatures. Because the intrinsic resistivity at low temperature is probably nearly isotropic, the changes in resistivity at these temperatures are most likely due to changes in magnetic disorder driven by domain reversal.

No steplike feature in $\rho(\varepsilon)$ is resolvable above $\sim 9 \mathrm{~K}$ for either stress orientation, despite the clear indication from neutron data of polarizable domains up to $T_{N}$. In principle, it is possible that domains are strongly pinned and do not reorient in strain ramps. Therefore we also performed temperature ramps from above $T_{N}$ down to $25.5 \mathrm{~K}$, conditions under which the neutron data indicate unambiguously that the magnetic order polarizes under strains well below $10^{-3}$ for any plausible assumption about the Young's modulus of $\mathrm{PdCrO}_{2}$. Again, there is no resolvable step at or near $\varepsilon=0$ [see Fig. 5(a)]. We conclude that any intrinsic resistive anisotropy within a domain is below our resolution.
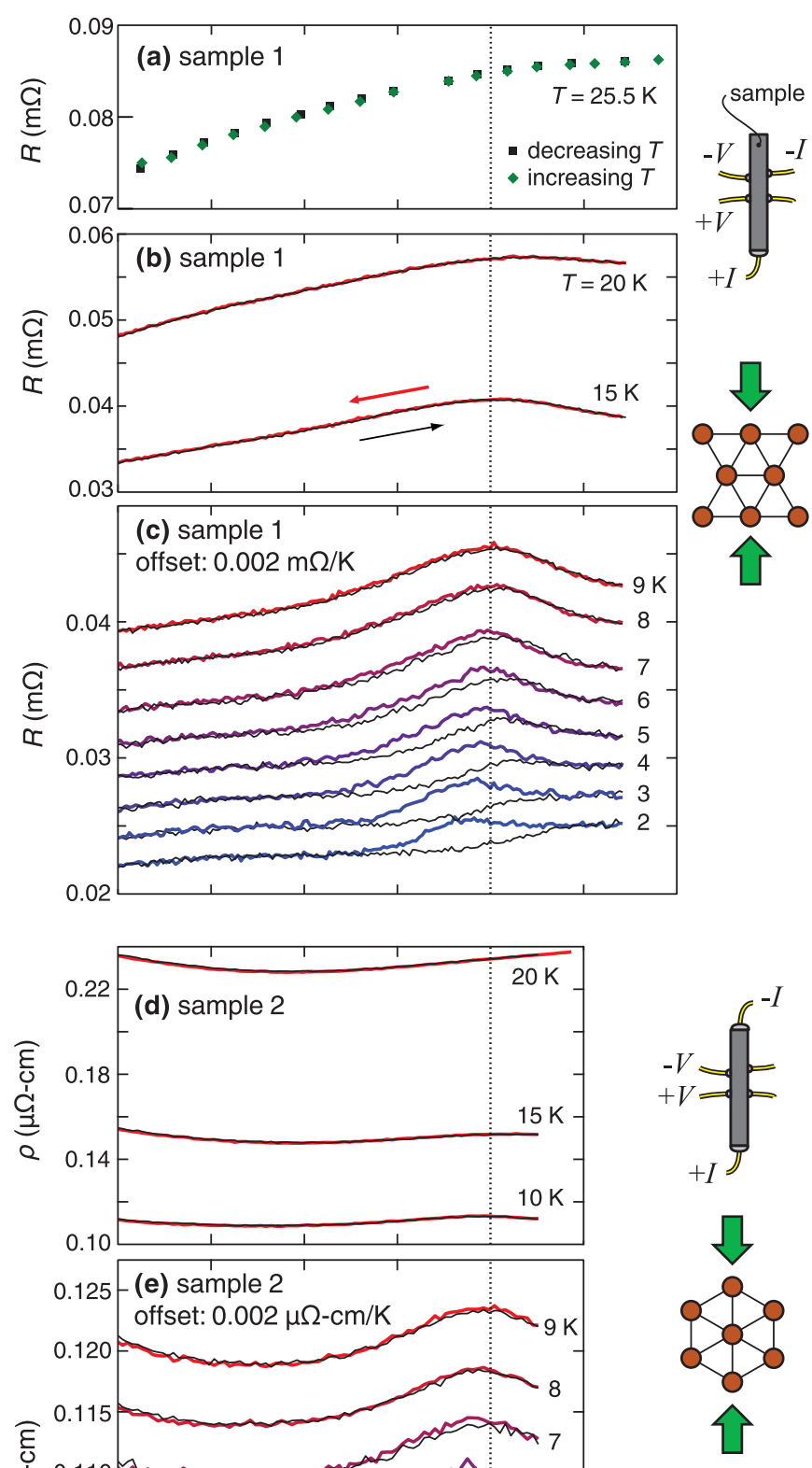

FIG. 5. Resistance versus strain of two samples of $\mathrm{PdCrO}_{2}$. (a)-(c) A sample cut along a $\langle 1 \overline{1} 0\rangle$ direction. One of the contacts broke during cooling — the sample was less than $1 \mathrm{~mm}$ long-so we used the contact configuration indicated. Because of the irregular contact configuration, data are plotted in units of resistance rather than resistivity. Data in (a) are from temperature ramps at fixed strain from above $T_{N}$, and data in (b) and (c) are from strain ramps at fixed temperature. (d) and (e) A sample cut along a $\langle 100\rangle$ direction. For all panels, $\varepsilon=0$ is taken as the location of the peak in $\rho$ at $9 \mathrm{~K}$. Colored lines are decreasing-strain ramps; black lines are increasing-strain ramps. The ramp rate for all curves is below $10^{-6} \mathrm{~s}^{-1}$. 
Strain does, however, have a strong effect on $\rho$ : the presence of the peak at $\varepsilon=0$, especially prominent for $\sim 4<$ $T<15 \mathrm{~K}$, indicates that there is magnetic disorder that can be suppressed by uniaxial stress. The peak broadens as $T$ is raised, indicating that it is thermal disorder. The sharpness of the peak at lower temperatures is striking. Even though the magnetic structure lifts the triangular symmetry of the lattice, it appears that being close to triangular symmetry gives a high susceptibility to thermal disorder, through the existence of one or more low-energy spin wave modes.

\section{RESULTS: MODELING}

In order to determine which degrees of freedom in the magnetic structure could give thermal disorder that is suppressed by uniaxial stress, we calculate scattering intensities for magnetic structures that deviate from that shown in Fig. 2(a). Following previous work $[6,16]$, the order within each layer is taken to be coplanar $120^{\circ}$ order, favored by strong intralayer interactions, and $\phi_{i}$ and $\alpha_{i}$ are the polar and azimuthal angles, respectively, of a selected spin within layer $i$. There is formally a third degree of freedom, $\beta_{i}$, the azimuthal angle of the spin plane about this reference spin, which sets the orientations of the other two spins; however, $\alpha$ and $\beta$ become indistinguishable parameters in the limit $\phi \rightarrow 0$. We calculate a goodness of fit $S^{\prime}=\left[\chi^{2} /(n-1)\right]^{1 / 2}$ to the scattering intensities reported in Ref. [16], where $n=58$ is the number of reported intensities and 1 is subtracted to account for an overall scaling factor to match the calculated to the measured intensities. As in that reference, we average over the three domain types, making the assumption that they are equally populated. $S^{\prime}$ below $\sim 1.3$ indicates a good fit. The magnetic structure shown in Fig. 2(a), which has $\phi=16^{\circ}$ and $\alpha=\beta=0$ in each layer, gives $S^{\prime}=1.06$. Full calculation results are given in the Appendix. The refinement in model 3 of Ref. [16] is layer-to-layer variation in $\phi$, and that in model 4 is layer-to-layer variation in $\alpha$. These refinements give $S^{\prime}=1.00$ and 0.99 , respectively, indicating only a minor improvement in the fit to data from our simplified, co-planar model.

$\phi=16^{\circ}$ is small enough that the distinction between $\alpha$ and $\beta$ is not very meaningful, and so we now fix $\beta=0$ and allow rotations of the spins out of the (110) plane only through nonzero $\alpha$. We consider nonzero $\beta$ in the Appendix. In Ref. [6], a spin wave gap of $0.4 \mathrm{meV}$ was observed in inelastic neutron scattering and reproduced in calculations of the in-plane dipolar anisotropy based on classical dipoledipole interactions. The value of $0.4 \mathrm{meV}$ corresponds to a temperature of $\sim 4 \mathrm{~K}$, which is approximately the temperature at which a peak in $\rho(\varepsilon)$ becomes discernible. Uniaxial stress will directly alter the in-plane anisotropy of magnetic interactions. It therefore appears likely that the effect of uniaxial stress is to increase the spin wave gap for spin excitations out of the (110) plane, reducing the amount of magnetic scattering and so also the resistivity.

To probe how far spins might fluctuate away from the (110) plane, we calculate intensities from a 300-layer magnetic cell within which $\alpha$ is chosen randomly in each layer, from a Gaussian distribution centered on $\alpha=0$ and with standard deviation $\sigma_{\alpha}$. At $2 \mathrm{~K}$, the best match to observed intensities is obtained when $\sigma_{\alpha}=10^{\circ}-15^{\circ}$. However, the improvement on locking the spins into the (110) plane is marginal: $S^{\prime}$ decreases from 1.06 to 0.97 . At $30 \mathrm{~K}$, however, the best match is obtained with $\sigma_{\alpha} \approx 20^{\circ}$, and now $S^{\prime}$ decreases from 1.30 to 1.10 . Therefore the neutron data of Ref. [16] suggest with modest statistical confidence that in unstressed $\mathrm{PdCrO}_{2}$ the fluctuation amplitude in $\alpha$ increases more rapidly than in $\phi$ as temperature is raised, in other words that the magnetic order softens more rapidly through fluctuations of spins out of the (110) plane than through other spin wave modes. We note that a calculation with $\alpha$ alternating regularly from layer to layer gives statistically indistinguishable results; however, a hypothesis of random variation is more consistent with thermal disorder.

\section{CONCLUSION}

In conclusion, we have shown through neutron scattering measurements under applied uniaxial stress and applied magnetic field that the magnetic order of $\mathrm{PdCrO}_{2}$ relieves interlayer frustration by spontaneously breaking the threefold rotational symmetry of the nonmagnetic lattice. By studying polarized magnetic structures, we also show that, as has been previously hypothesized, the double- $k$ nature of the magnetic structure is intrinsic, due to alternating helicity from layer to layer within each domain, rather than a consequence of mixture of different magnetic structures. The rotational symmetry breaking is not detectable in resistivity, consistent with it being driven by magnetic interactions between the $\mathrm{Cr}$ sites rather than an electronic susceptibility in the Pd sheets. Resistivity measurements indicate the presence of low-energy spin wave modes when the lattice is close to triangularly symmetric. At low temperatures, these result in a pronounced peak in sample resistivity near zero applied stress due to magnetic disorder, that can be suppressed by increasing the applied stress. More generally, the ability to polarize magnetic domains through uniaxial stress will in the future allow greater precision in the determination of magnetic structures, by eliminating domain population as a degree of freedom.

The datasets for the neutron experiments are available from the Science and Technology Facilities Council ISIS Neutron and Muon Source Data Journal [33,34], and transport data presented in Figs. 5 and 9 are available at [35].

\section{ACKNOWLEDGMENTS}

We acknowledge helpful discussions with E. Berg, T. Oka, and O. Erten. We acknowledge the financial support of the Max Planck Society. Experiments at the ISIS Pulsed Neutron and Muon Source were supported by a beam time allocation from the Science and Technology Facilities Council under Expt. No. RB1520411, for the field work and Expt. No. RB1800029, for the stress work. P.K. thanks SERB-DST, India for Ramanujan Fellowship (Award No: SB/S2/RJN091/2017). 


\section{APPENDIX}

\section{Measurement setup for neutron scattering}

Figure 6 illustrates the uniaxial stress apparatus that we used here for neutron scattering. A platelike sample geometry is compatible with application of very high uniaxial stress, with high stress homogeneity. In our apparatus, samples are held in detachable holders (allowing rapid sample exchange during beam time) that leave as much space around the sample (a)
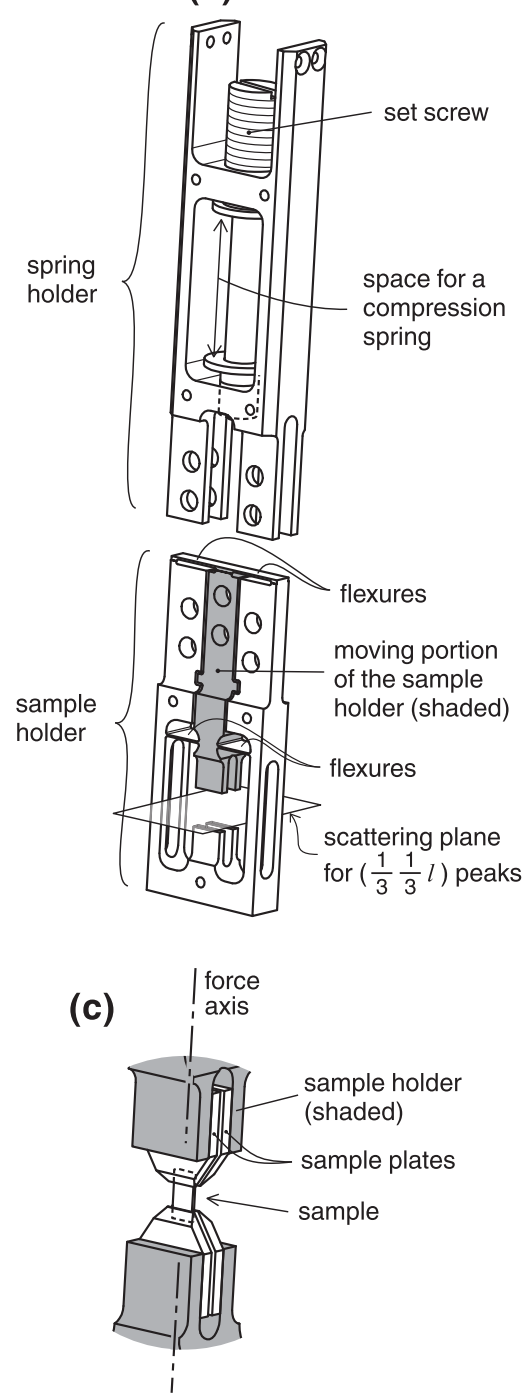

(b)

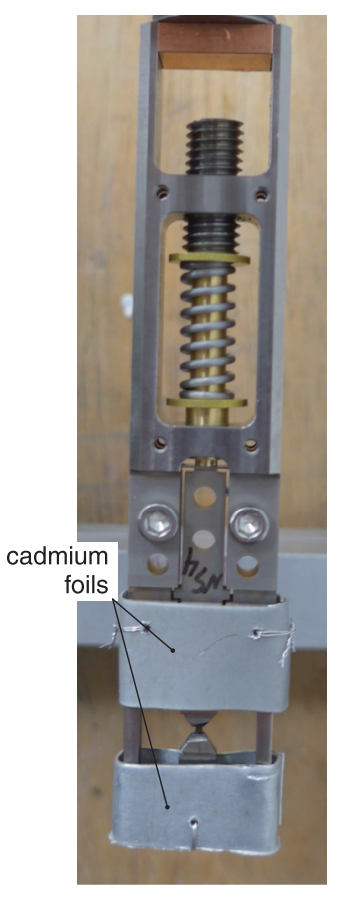

(d)

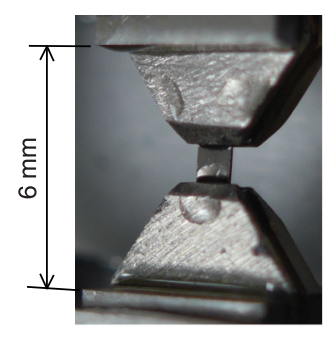

FIG. 6. The experiment setup for neutron scattering. (a) Drawing of the spring and sample holders. The configuration of the spring holder in this drawing is for applying compressive load onto the sample; however, a tension spring can also be installed. Force is applied to a moving portion of the sample holder, whose motion is constrained by flexures to be longitudinal with respect to the sample. This protects the sample from inadvertent transverse or twisting forces. (b) Photograph of this setup, including cadmium foils used to absorb stray neutrons. (c) Drawing of a mounted sample, with the force axis indicated. The sample is platelike, and each end is secured with epoxy between two sample plates. The end portions of the sample, embedded in the epoxy, will be under lower stress than the central, exposed portion. (d) A photograph of sample A, taken shortly after removal from the cryostat. exposed as possible. The holder incorporates flexures that protect the sample from inadvertent twisting or transverse forces; this is essential because the samples are thin and mechanically fragile. The holder slots into a spring holder, which holds either a compression or tension spring to apply force to the sample. A set screw is used to adjust the force; the force applied was determined by multiplying the spring constant of the spring, supplied by the manufacturer, by the applied displacement, which was measured with a ruler. As the set screw can be adjusted only at room temperature, samples are cooled under approximately constant stress. The spring constant of the springs used will have increased by $\sim 10 \%$ with cooling to cryogenic temperatures, introducing some uncertainty in the uniaxial stress achieved at low $T$, however this does not affect any of our conclusions.

The two samples of $\mathrm{PdCrO}_{2}$ probed with neutron scattering were oriented using a backscattering X-ray Laue instrument, ground with silicon carbide lapping film to thicknesses of 0.07 and $0.11 \mathrm{~mm}$, and cut along the [110] direction using a commercial wire saw. They were secured in the sample holders using Stycast 2850FT epoxy.

\section{Calculation of scattering intensities}

The magnetic reflections are indexed to a three-site nonmagnetic unit cell. The lattice vectors of this cell are

$$
\begin{aligned}
& \mathbf{a}=\left(\frac{a}{2},-\frac{a \sqrt{3}}{2}, 0\right), \\
& \mathbf{b}=\left(\frac{a}{2}, \frac{a \sqrt{3}}{2}, 0\right), \\
& \mathbf{c}=(0,0, c) .
\end{aligned}
$$

Here $a=2.93 \AA$ is the $\mathrm{Cr}-\mathrm{Cr}$ interatomic spacing, and $c=$ $18.087 \AA$ spans three layers. The reciprocal lattice vectors of this cell are

$$
\begin{aligned}
& \mathbf{h}_{3}=2 \pi\left(\frac{1}{a},-\frac{1}{a \sqrt{3}}, 0\right), \\
& \mathbf{k}_{\mathbf{3}}=2 \pi\left(\frac{1}{a}, \frac{1}{a \sqrt{3}}, 0\right), \\
& \mathbf{l}_{\mathbf{3}}=2 \pi\left(0,0, \frac{1}{c}\right) .
\end{aligned}
$$

The atomic positions in the first layer are given by

$$
\begin{aligned}
& \mathbf{x}_{1}=(0,0,0), \\
& \mathbf{x}_{2}=\left(\frac{a}{2}, \frac{a \sqrt{3}}{2}, 0\right), \\
& \mathbf{x}_{3}=\left(-\frac{a}{2}, \frac{a \sqrt{3}}{2}, 0\right) .
\end{aligned}
$$

The magnetic moments on these sites are given by

$$
\begin{aligned}
& \mathbf{M}_{1}=\left(\sin \left(\phi_{1}\right) \sin \left(\alpha_{1}\right), \sin \left(\phi_{1}\right) \cos \left(\alpha_{1}\right), \cos \left(\phi_{1}\right)\right), \\
& \mathbf{M}_{2}=\cos \left(\frac{2 \pi}{3}\right) \mathbf{M}_{1}+\sin \left(\frac{2 \pi}{3}\right) \mathbf{M}_{1} \times \hat{\mathbf{r}}, \\
& \mathbf{M}_{3}=\cos \left(-\frac{2 \pi}{3}\right) \mathbf{M}_{1}+\sin \left(-\frac{2 \pi}{3}\right) \mathbf{M}_{1} \times \hat{\mathbf{r}},
\end{aligned}
$$


where

$$
\begin{aligned}
\hat{\mathbf{r}}= & \left(\cos \left(\beta_{1}\right) \cos \left(\alpha_{1}\right)-\sin \left(\beta_{1}\right) \sin \left(\alpha_{1}\right) \cos \left(\phi_{1}\right),\right. \\
& -\cos \left(\beta_{1}\right) \sin \left(\alpha_{1}\right)-\sin \left(\beta_{1}\right) \cos \left(\alpha_{1}\right) \cos \left(\phi_{1}\right), \\
& \left.\sin \left(\beta_{1}\right) \sin \left(\phi_{1}\right)\right) .
\end{aligned}
$$

The spin rotation angles $\phi, \alpha$, and $\beta$ are illustrated in Fig. 2; $\phi$ and $\alpha$ are, respectively, the polar and azimuthal angles of a reference spin in each layer, and $\beta$ is the azimuthal angle of the spin plane about this reference spin. In the above expressions, the subscript on $\phi, \alpha$, and $\beta$ refers to the layer number. $\alpha$ is defined to be zero when the reference spin lies in the (110) plane, and $\beta$ is defined to be zero when the spin plane contains the [001] axis.

The atomic positions and magnetic moment orientations in subsequent layers are taken as illustrated in Fig. 2. To calculate scattering intensities, we sum over the 18 sites of the magnetic unit cell:

$$
\mathbf{M}(\mathbf{q})=\sum_{i=1}^{18} e^{i \mathbf{q} \cdot \mathbf{R}_{i}} \mathbf{M}_{i} .
$$
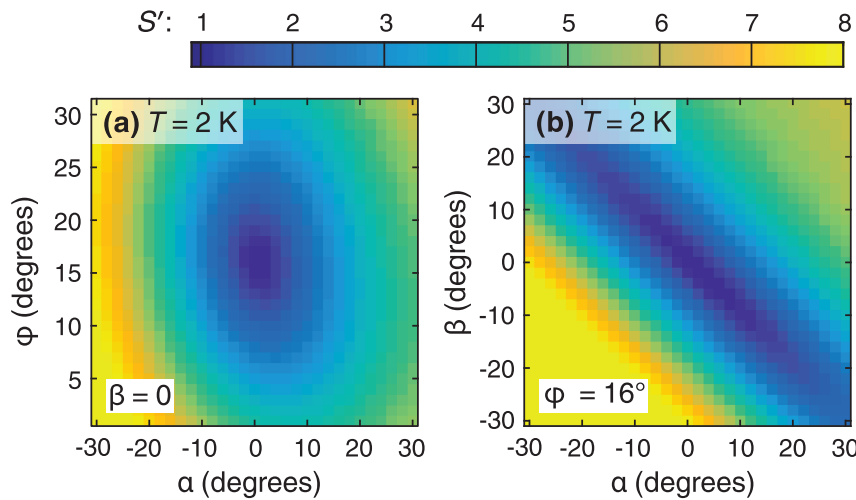

$\begin{array}{llllllllllll}S^{\prime}: & 1.0 & 1.2 & 1.4 & 1.6 & 1.8 & 2.0 & 2.2 & 2.4 & 2.6 & 2.8 & 3.0\end{array}$
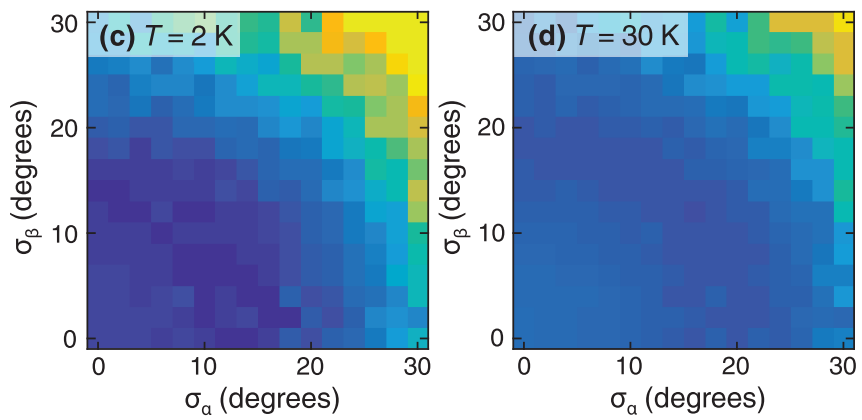

FIG. 7. (a) and (b) Goodness of fit $S^{\prime}$ to the scattering intensities at $2 \mathrm{~K}$ reported in Ref. [16] for magnetic structures with identical $\phi$, $\alpha$, and $\beta$ in each layer. In (a), $\beta$ is set to zero, and in (b) $\phi$ is set to $16^{\circ}$. (c) and (d) Goodness of fit $S^{\prime}$ to the scattering intensities at 2 and $30 \mathrm{~K}$, respectively, reported in Ref. [16], taking $\phi=16^{\circ}$ in each layer and allowing $\alpha$ and $\beta$ to vary randomly from layer to layer, drawn from Gaussian distributions centered on $0^{\circ}$ and with standard deviations $\sigma_{\alpha}$ and $\sigma_{\beta}$.
Neglecting prefactors, the scattering intensities are

$$
I(\mathbf{q})=f(|\mathbf{q}|) \times\left|\mathbf{M}_{\perp}(\mathbf{q})\right|^{2},
$$

where $\mathrm{f}(\mathbf{q})$ is the magnetic form factor of $\mathrm{Cr}^{3+}$ and $\mathbf{M}_{\perp}(\mathbf{q})=$ $\mathbf{M}(\mathbf{q})-\hat{\mathbf{q}}(\hat{\mathbf{q}} \cdot \mathbf{M})$. The goodness of fit $S^{\prime}$ is given by

$$
S^{\prime}=\left[\frac{1}{57} \sum_{i=1}^{58}\left(\frac{S_{\mathrm{obs}, i}-x S_{\mathrm{calc}, i}}{\sigma_{i}}\right)^{2}\right]^{1 / 2},
$$

where $x=\sum S_{\text {obs }} / \sum S_{\text {calc }}$ and 58 is the number of intensities reported in Ref. [11]. The magnetic structure, as explained in the main text, lifts the threefold rotational symmetry of the nonmagnetic lattice, and so to compare with the intensities reported in Ref. [11] we average over the three possible rotations of this structure about $\hat{\mathbf{z}}$.

In Figs. 7(a) and 7(b) we show $S^{\prime}$ for a magnetic structure in which $\phi, \alpha$, and $\beta$ are the same in each layer. $\phi$ is seen to be tightly constrained: deviation from $16^{\circ}$ by more than $3^{\circ}$ raises $S^{\prime}$ above 1.3. $\alpha$ and $\beta$ become indistinguishable parameters in the limit $\phi \rightarrow 0$, and $\phi=16^{\circ}$ is small enough that, as illustrated in Fig. 7(b), $\alpha$ and $\beta$ are not tightly constrained mathematically. However, the sum $\alpha+\beta$ is tightly constrained around zero, indicating that the best fit is obtained when the spins lie within the (110) plane.

In Figs. 7(c) and 7(d) we illustrate $S^{\prime}$ for a model where layer-to-layer fluctuations of the spins out of the (110) plane are allowed. This is the model described in the main text: a 300-layer magnetic unit cell is taken, $\phi$ is fixed at $16^{\circ}$ in all layers, and $\alpha$ and $\beta$ in each layer are drawn randomly from Gaussian probability distributions centered on $0^{\circ}$ with standard deviations $\sigma_{\alpha}$ and $\sigma_{\beta}$. Because $\alpha$ and $\beta$ are not highly distinct parameters for small $\phi, S^{\prime}$ is found to be nearly constant along lines of constant $\sqrt{\sigma_{\alpha}^{2}+\sigma_{\beta}^{2}}$. As described in

TABLE I. Integrated intensities of $\left(\frac{1}{3}, \frac{1}{3}, l\right)$ magnetic reflections measured at $2 \mathrm{~K}$ under various compressive stresses. Because the temperature-independent background was measured only at $-60 \mathrm{MPa}$, here we do not subtract any background. The reported intensities of each reflection have been normalized by the incident neutron flux at that wavelength.

\begin{tabular}{lcccc}
\hline \hline \multicolumn{5}{c}{ Stress } \\
\hline$(h, k, l)$ & $0 \mathrm{MPa}$ & $-24 \mathrm{MPa}$ & $-60 \mathrm{MPa}$ & $-108 \mathrm{MPa}$ \\
\hline$\left(\frac{1}{3}, \frac{1}{3},-1\right)$ & $14.2(5)$ & $19.9(4)$ & $23.5(4)$ & $28.5(4)$ \\
$\left(\frac{1}{3}, \frac{1}{3},-0.5\right)$ & $12.0(4)$ & $15.2(3)$ & $16.0(3)$ & $15.3(3)$ \\
$\left(\frac{1}{3}, \frac{1}{3}, 0\right)$ & $19.2(7)$ & $13.7(4)$ & $3.9(2)$ & $4.0(2)$ \\
$\left(\frac{1}{3}, \frac{1}{3}, 0.5\right)$ & $7.6(5)$ & $9.8(4)$ & $12.4(4)$ & $14.2(4)$ \\
$\left(\frac{1}{3}, \frac{1}{3}, 1\right)$ & $26(1)$ & $32.5(8)$ & $33.7(8)$ & $31.0(7)$ \\
$\left(\frac{1}{3}, \frac{1}{3}, 1.5\right)$ & $16(1)$ & $11.5(6)$ & $3.4(3)$ & $4.1(3)$ \\
$\left(\frac{1}{3}, \frac{1}{3}, 2\right)$ & $8.4(8)$ & $8.2(5)$ & $11.8(6)$ & $18.9(6)$ \\
$\left(\frac{1}{3}, \frac{1}{3}, 2.5\right)$ & $6.6(7)$ & $8.5(5)$ & $9.1(5)$ & $2.1(2)$ \\
$\left(\frac{1}{3}, \frac{1}{3}, 3\right)$ & $9.4(8)$ & $7.9(5)$ & $2.9(3)$ & $1(1)$ \\
$\left(\frac{1}{3}, \frac{1}{3}, 3.5\right)$ & $6.1(6)$ & $9.0(5)$ & $6.9(4)$ & $12.8(5)$ \\
$\left(\frac{1}{3}, \frac{1}{3}, 4\right)$ & $9.2(7)$ & $10.9(5)$ & $11.9(5)$ & $13.6(5)$ \\
\hline \hline
\end{tabular}



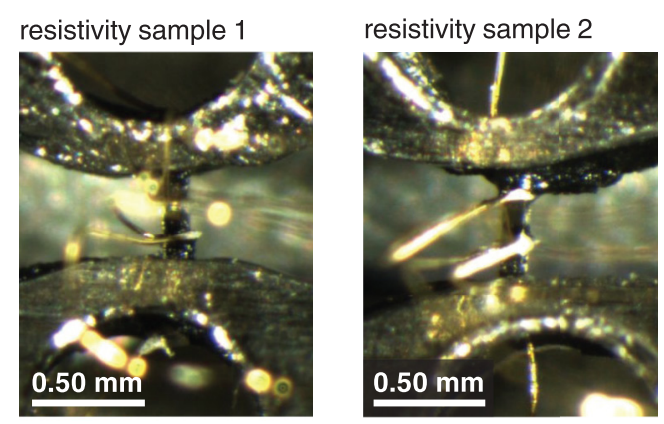

FIG. 8. Photographs of the two resistivity samples reported in Fig. 5; the left-hand photograph is of the $\langle 1 \overline{1} 0\rangle$ sample, and the right-hand photograph shows the $\langle 100\rangle$ sample.

the main text, at $2 \mathrm{~K}$ the best fit is obtained when the spins fluctuate by $\sim 12^{\circ}$ out of the (110) plane, but the improvement on locking them into the (110) plane $(\alpha=\beta=0)$ is not large: $S^{\prime}$ decreases from 1.06 to about 0.97 . At $30 \mathrm{~K}$ the best fit is obtained with $\sqrt{\sigma_{\alpha}^{2}+\sigma_{\beta}^{2}} \sim 20^{\circ}$, and now the improvement on $\alpha=\beta=0$ is larger: $S^{\prime}$ decreases from 1.30 to 1.11 . In other words, as temperature is raised the magnetic order appears to soften more rapidly through fluctuations of the spin directions out of the (110) plane than through fluctuations in other spin wave modes.

\section{Further data}

The integrated intensities of the $\left(\frac{1}{3}, \frac{1}{3}, l\right)$ magnetic reflections from sample $\mathrm{A}$ at $2 \mathrm{~K}$ are given in Table I.

Photographs of the two resistivity samples reported in Fig. 5 are shown in Fig. 8.

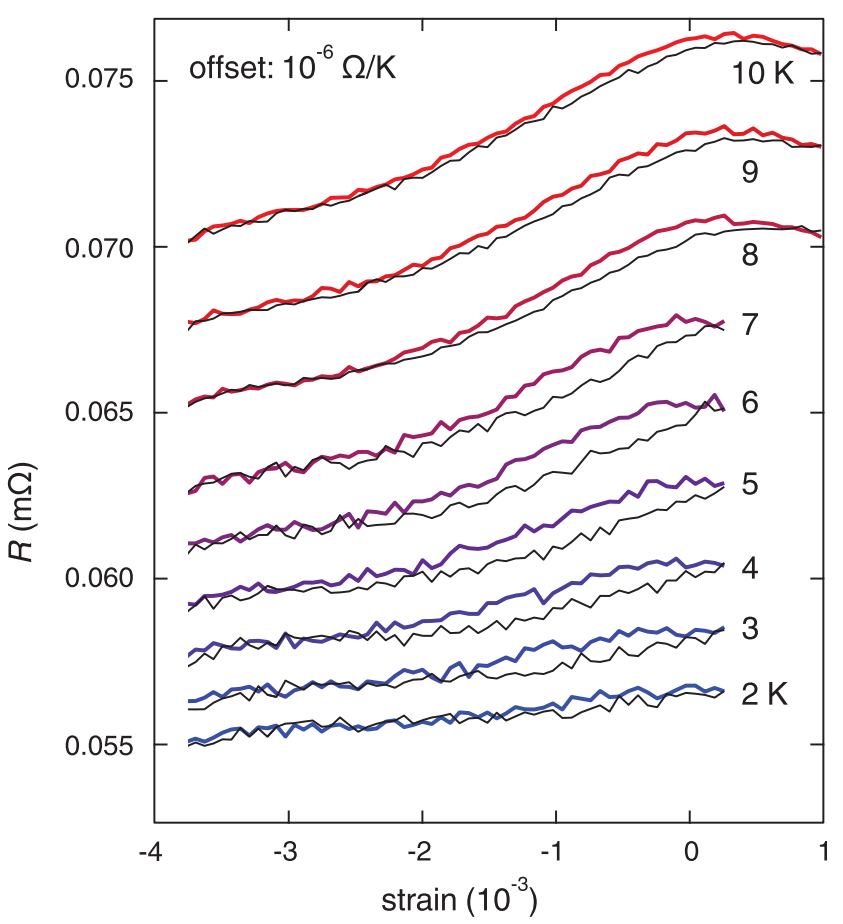

FIG. 9. Resistivity $\rho$ versus strain for a third resistivity sample. Pressure is applied along a $\langle 1 \overline{1} 0\rangle$ direction.

Figure 9 shows resistivity versus strain data for a third resistivity sample. Pressure was applied along a $\langle 1 \overline{1} 0\rangle$ direction, the same as for sample 1 in Fig. 5. In this sample, there is discernible hysteresis up to $10 \mathrm{~K}$. The hysteresis gradually flattens as $T$ is raised but at all temperatures extends out to a rather large strain of almost $3 \times 10^{-3}$.
[1] G. C. De Fotis, Magnetism of solid oxygen, Phys. Rev. B 23, 4714 (1981).

[2] P. W. Stephens and C. F. Majkrzak, Magnetic structure and dynamics in the $\alpha$ and $\beta$ phases of solid oxygen, Phys. Rev. B 33, 1 (1986).

[3] E. Rastelli and A. Tassi, Collinear and helical phases of the monoclinic and rhombohedral antiferromagnet. Application to the $\alpha$ - and $\beta$-phases of solid oxygen, J. Phys. C 21, 1003 (1988).

[4] H. Takatsu, H. Yoshizawa, S. Yonezawa, and Y. Maeno, Critical behavior of the metallic triangular-lattice Heisenberg antiferromagnet $\mathrm{PdCrO}_{2}$, Phys. Rev. B 79, 104424 (2009).

[5] C. W. Hicks, A. S. Gibbs, L. Zhao, P. Kushwaha, H. Borrmann, A. P. Mackenzie, H. Takatsu, S. Yonezawa, Y. Maeno, and E. A. Yelland, Quantum oscillations and magnetic reconstruction in the delafossite $\mathrm{PdCrO}_{2}$, Phys. Rev. B 92, 014425 (2015).

[6] M. D. Le, S. Jeon, A. I. Kolesnikov, D. J. Voneshen, A. S. Gibbs, J. S. Kim, J. Jeong, H.-J. Noh, C. Park, J. Yu, T. G. Perring, and J.-G. Park, Magnetic interactions in $\mathrm{PdCrO}_{2}$ and their effects on its magnetic structure, Phys. Rev. B 98, 024429 (2018).

[7] S. Arsenijević, J. M. Ok, P. Robinson, S. Ghannadzadeh, M. I. Katsnelson, J. S. Kim, and N. E. Hussey, Anomalous Magne- tothermopower in a Metallic Frustrated Antiferromagnet, Phys. Rev. Lett. 116, 087202 (2016).

[8] D. Billington, D. Ernsting, T. E. Millichamp, C. Lester, S. B. Dugdale, D. Kersh, J. A. Duffy, S. R. Giblin, J. W. Taylor, P. Manuel, D. D. Khalyavin, and H. Takatsu, Magnetic frustration, short-range correlations and the role of the paramagnetic Fermi surface of $\mathrm{PdCrO}_{2}$, Sci. Rep. 5, 12428 (2015).

[9] H. Takatsu, S. Yonezawa, C. Michioka, K. Yoshimura, and Y. Maeno, Anisotropy in the magnetization and resistivity of the metallic triangular-lattice magnet $\mathrm{PdCrO}_{2}$, J. Phys.: Conf. Ser. 200, 012198 (2010).

[10] K. Kimura, H. Nakamura, S. Kimura, M. Hagiwara, and T. Kimura, Tuning Ferroelectric Polarization Reversal by Electric and Magnetic Fields in $\mathrm{CuCrO}_{2}$, Phys. Rev. Lett. 103, 107201 (2009).

[11] M. Soda, K. Kimura, T. Kimura, and K. Hirota, Domain rearrangement and spin-spiral-plane flop as sources of magnetoelectric effects in delafossite $\mathrm{CuCrO}_{2}$, Phys. Rev. B 81, 100406(R) (2010).

[12] M. Frontzek, G. Ehlers, A. Podlesnyak, H. Cao, M. Matsuda, O. Zaharko, N. Aliouane, S. Barilo, and S. V. Shiryaev, Magnetic structure of $\mathrm{CuCrO}_{2}$ : A single crystal neutron diffraction study, J. Phys.: Condens. Matter 24, 016004 (2012). 
[13] Y. A. Sakhratov, L. E. Svistov, P. L. Kuhns, H. D. Zhou, and A. P. Reyes, Magnetic Structure and Domain Conversion of the Quasi-2D Frustrated Antiferromagnet $\mathrm{CuCrO}_{2}$ Probed by NMR, J. Exp. Theor. Phys. 119, 880 (2014).

[14] S. Seki, Y. Onose, and Y. Tokura, Spin-Driven Ferroelectricity in Triangular Lattice Antiferromagnets $A \mathrm{CrO}_{2}(A=\mathrm{Cu}, \mathrm{Ag}, \mathrm{Li}$, or Na), Phys. Rev. Lett. 101, 067204 (2008).

[15] O. Aktas, G. Quirion, T. Otani, and T. Kimura, First-order ferroelastic transition in a magnetoelectric multiferroic: $\mathrm{CuCrO}_{2}$, Phys. Rev. B 88, 224104 (2013).

[16] H. Takatsu, G. Nénert, H. Kadowaki, H. Yoshizawa, M. Enderle, S. Yonezawa, Y. Maeno, J. Kim, N. Tsuji, M. Takata, Y. Zhao, M. Green, and C. Broholm, Magnetic structure of the conductive triangular-lattice antiferromagnet $\mathrm{PdCrO}_{2}, \mathrm{Phys}$. Rev. B 89, 104408 (2014).

[17] A. P. Mackenzie, The properties of ultrapure delafossite metals, Rep. Prog. Phys. 80, 032501 (2017).

[18] J.-H. Chu, J. G. Analytis, K. De Greve, P. L. McMahon, Z. Islam, Y. Yamamoto, and I. R. Fisher, In-plane resistivity anisotropy in an underdoped iron arsenide superconductor, Science 329, 824 (2010).

[19] V. Sunko, F. Mazzola, S. Kitamura, S. Khim, P. Kushwaha, O. J. Clark, M. Watson, I. Marković, D. Biswas, L. Pourovskii, T. K. Kim, T.-L. Lee, P. K. Thakur, H. Rosner, A. Georges, R. Moessner, T. Oka, A. P. Mackenzie, and P. D. C. King, Probing spin correlations using angle resolved photoemission in a coupled metallic/Mott insulator system, arXiv:1809.08972.

[20] J. M. Ok, Y. J. Jo, K. Kim, T. Shishidou, E. S. Choi, H.-J. Noh, T. Oguchi, B. I. Min, and J. S. Kim, Quantum Oscillations of the Metallic Triangular-Lattice Antiferromagnet $\mathrm{PdCrO}_{2}$, Phys. Rev. Lett. 111, 176405 (2013).

[21] H.-J. Noh, J. Jeong, B. Chang, D. Jeong, H. S. Moon, E.-J. Cho, J. M. Ok, J. S. Kim, K. Kim, B. I. Min, H.-K. Lee, J.-Y. Kim, B.-G. Park, H.-D. Kim, and $\mathrm{S}$. Lee, Direct observation of localized spin antiferromagnetic transition in $\mathrm{PdCrO}_{2}$ by angle-resolved photoemission spectroscopy, Sci. Rep. 4, 3680 (2014).

[22] R. Daou, R. Frésard, S. Hébert, and A. Maignan, Impact of short-range order on transport properties of the twodimensional metal $\mathrm{PdCrO}_{2}$, Phys. Rev. B 92, 245115 (2015).

[23] S. Ghannadzadeh, S. Licciardello, S. Arsenijević, P. Robinson, H. Takatsu, M. I. Katsnelson, and N. E. Hussey, Simultaneous loss of interlayer coherence and long-range magnetism in quasitwo-dimensional $\mathrm{PdCrO}_{2}$, Nat. Commun. 8, 15001 (2017).
[24] In Ref. [32], an anomalous Hall effect was reported in $\mathrm{PdCrO}_{2}$ in the absence of resolvable magnetization, which was interpreted as indicating nonzero scalar spin chirality, in other words noncoplanar spins. This anomalous Hall effect, however, is induced by a nonzero field, so we think that the possibility that the spins are coplanar in zero field should not be excluded.

[25] H. Takatsu and Y. Maeno, Single crystal growth of the metallic triangular-lattice antiferromagnet $\mathrm{PdCrO}_{2}$, J. Cryst. Growth 312, 3461 (2010).

[26] J. Paglione, C. Lupien, W. A. MacFarlane, J. M. Perz, L. Taillefer, Z. Q. Mao, and Y. Maeno, Elastic tensor of $\mathrm{Sr}_{2} \mathrm{RuO}_{4}$, Phys. Rev. B 65, 220506(R) (2002).

[27] J. J. Roa, X. G. Capdevila, M. Martínez, F. Espiell, and M. Segarra, Nanohardness and Young's modulus of YBCO samples textured by the Bridgman technique, Nanotechnology 18, 385701 (2007).

[28] J. A. Sobota, K. Kim, H. Takatsu, M. Hashimoto, S.-K. Mo, Z. Hussain, T. Oguchi, T. Shishidou, Y. Maeno, B. I. Min, and Z.-X. Shen, Electronic structure of the metallic antiferromagnet $\mathrm{PdCrO}_{2}$ measured by angle-resolved photoemission spectroscopy, Phys. Rev. B 88, 125109 (2013).

[29] C. W. Hicks, M. E. Barber, S. D. Edkins, D. O. Brodsky, and A. P. Mackenzie, Piezoelectric-based apparatus for strain tuning, Rev. Sci. Inst. 85, 065003 (2014).

[30] M. E. Barber, A. S. Gibbs, Y. Maeno, A. P. Mackenzie, and C. W. Hicks, Resistivity in the Vicinity of a van Hove Singularity: $\mathrm{Sr}_{2} \mathrm{RuO}_{4}$ under Uniaxial Pressure, Phys. Rev. Lett. 120, 076602 (2018).

[31] M. E. Barber, A. Steppke, A. P. Mackenzie, and C. W. Hicks, Piezoelectric-based uniaxial pressure cell with integrated force and displacement sensors, Rev. Sci. Instrum. 90, 023904 (2019).

[32] H. Takatsu, S. Yonezawa, S. Fujimoto, and Y. Maeno, Unconventional Anomalous Hall Effect in the Metallic TriangularLattice Magnet $\mathrm{PdCrO}_{2}$, Phys. Rev. Lett. 105, 137201 (2010).

[33] A. Gibbs et al., Data for "Low temperature structure and magnetostructural coupling in $\mathrm{PdCrO}_{2}$, STFC ISIS Neutron and Muon Source", https://doi.org/10.5286/ISIS.E.67774469 (2015).

[34] D. Sokolov et al., Data for "New apparatus for neutron diffraction under uniaxial pressure, STFC ISIS Neutron and Muon Source", https://doi.org/10.5286/ISIS.E.90605228 (2018).

[35] https://dx.doi.org/10.17617/3.2s or https://edmond.mpdl.mpg. de/imeji/collection/s2o5aaHTBB1dhpZP. 